\title{
Safety \& Efficacy of Propranolol and Amitriptyline Combination Therapy in Migraine Prophylaxis: A Randomized Control Trial
}

\author{
Mohammad Ariful Islam', Abdul Kader Shaikh², Sk. Mahbub Alam³, \\ Dahlia Sultana ${ }^{4}$, Md. Saiful Islam ${ }^{5}$, Md. Mamnur Rashid ${ }^{6}$
}

\begin{abstract}
${ }^{1}$ Assistant Professor, Department of Neurology, National Institute of Neurosciecnes\& Hospital, Dhaka, Bangladesh; ${ }^{2}$ Associate Professor, Department of Neurology, Bangabandhu Sheikh Mujib Medical University, Dhaka, Bangladesh; ${ }^{3}$ Associate Professor, Department of Neurology, Bangabandhu Sheikh Mujib Medical University, Dhaka, Bangladesh; ${ }^{4}$ Assistant Professor, Department of Endocrinology \& Metabolism, Sir Salimullah Medical College \& Mitford Hospital, Dhaka, Bangladesh; ${ }^{5}$ Research Officer, Directorate General of Health Services, Ministry of Health \& Family Welfare, Dhaka, Bangladesh; ${ }^{6}$ Resident Physician, Department of Neurology, National Institute of Neurosciecnes\& Hospital, Dhaka, Bangladesh;
\end{abstract}

[Received: 27 July 2017; Revised: 25 August 2017; Accepted: 30 September 2017; Published: 1 January 2018]

\begin{abstract}
Background: Combination of propranolol and amitriptyline drugs an be effective for migraine prophylaxis. Objective: The purpose of the present study was to see the safety and efficacy of propranolol and amitriptyline combination therapy in migraine prophylaxis. Methodology: This study randomized control trial was conducted in headache clinic at Banghabandhu Sheikh Medical University (BSMMU), Dhaka, Bangladesh from July 2012 to June 2014 for a period of two (02) years. Migraine patients with or without aura of 16 to 50 years of age, patients not on any prophylactic medication and patients willing to take part in the study were included for this study. Patients meeting all the criteria was randomized for two (02) treatment groups designated as the group A who were treated with Amitriptyline and the group B who were treated with the combination of amitriptyline and propranolol. Patients was followed for a three months period during which they were instructed to maintain a headache diary. The primary outcome evaluated was the proportion of patients in each group that achieved a $50 \%$ reduction in the number of days with headache. Secondary outcomes was reduction of visual analogue pain scale score, the number of days with headache per month, frequency of side effects and the proportion of patients abandoning the study before the end of medication. The causes of noncompliance and side effects was individually registered. Result: A total number of 8 0patietns were recruited for this study. During 1st visit among the patients in group A, duration of pain 1-4 hours $1(2.5 .0 \%)$, 5-8 hours $16(13.3 \%)$ and $9-12$ hours $14(35.0 \%)$. In group B, duration of pain 1-4 hours $0(0.0 \%), 5-8$ hours $18(15.0 \%) 9-12$ were $21(52.5 \%)$, above 13 hours pain duration were $1(2.5 \%)(\mathrm{p}>0.05)$. Duration of pain was recorded in final follow up among the patients. In group A, duration of pain 1-4 hours $24(60.0 \%), 5-8$ hours $14(35.0 \%)$, 9-12 hours $2(5.0 \%)$. In group B, duration of pain $1-4$ hours $28(70.0 \%), 5-8$ hours $12(30.0 \%), 9-12$ hours were not found $(\mathrm{p}>0.05)$. In group $\mathrm{A}$, no adverse effect was found $26(65.0 \%)$, drowsiness $6(15.0 \%)$, dryness of mouth $6(15.0 \%)$, constipation $2(5.0 \%)$, fatigue and bradycardia were not found. In group B, no adverse effect was found 29(72.5\%), drowsiness, dryness of mouth and constipation were not found, fatigue and bradycardia were $7(17.5 \%)$ and $4(10.0 \%)$. Number of attack and headache before treatment and subsequent follow up with medication it was found that number of attach and headache gradually decrease $(\mathrm{p}<0.05)$. Conclusion: In conclusion there is a significant changes of number of headache and attach in the amitriptyline and combine group. [Journal of National Institute of Neurosciences Bangladesh, 2018;4(1): 3-7]
\end{abstract}

\section{Keywords: Safety \& Efficacy of Propranolol and amitriptyline combination therapy in migraine prophylaxis}

Correspondence: Dr. Mohammad Ariful Islam, Assistant Professor, Department of Neurology, National Institute of Neurosciecnes \& Hospital, Dhaka, Bangladesh; Email: drariful22@gmail.com; Cell no.: +8801715016462

Conflict of interest: There is no conflict of interest relevant to this paper to disclose.

Funding agency: This research project was not funded by any group or any institution.

Contribution to authors: RFA \& JAcontributed from the protocol preparation up to report writing. JA involved in the manuscript writing and revision.

How to cite this article: Islam MA, Shaikh AK, AlamSkM, Sultana D, Islam MS, Rashid MM. Safety and Efficacy of Propranolol and amitriptyline combination therapy in migraine prophylaxis: A Randomized Control Trial. J NatlInstNeurosci Bangladesh, 2018;4(1): 3-7

Copyright: (C2018. Islam et al. Published by Journal of National Institute of Neurosciences Bangladesh. This article is published under the Creative Commons CC BY-NC License (https://creativecommons.org/licenses/by-nc/4.0/). This license permits use, distribution and reproduction in any medium, provided the original work is properly cited, and is not used for commercial purposes. 


\section{Introduction}

Migraine is now ranked by the World Health Organization as number 19 among all diseases causing disability world-wide ${ }^{1}$ (HCSIHS 2005). It is an episodic primary headache disorder that is characterized by recurrent attacks of various combinations of headache and neurological, gastrointestinal and autonomic symptoms (Charles 2009). World health organization (WHO) included the migraine in the Global burden of Disease study conducted in 2000 and reported in the world Health Report 2001. Successful management of migraine requires intensive patients' educations and thorough physician knowledge aboutavailable treatment options and strategies. Use of a prophylactic medication reduces headachefrequency, severity and risk for rebound (Leonardi et al. 2005).

Migraine is a common and disabling primary headache disorder with worldwide prevalence of $10-12 \%$ of adult population ${ }^{4}$. In Bangladesh there is no data regarding the prevalence of migraine. In a study conducted in BSMMU headache clinic total 3440 patients were studied and $16.05 \%$ of them had a diagnosis of migraine ${ }^{5}$. In another Bangladeshi study, the tension headache (Muscle contraction headache) was the commonest type (69\%) followed by migraine $(26 \%)^{6}$. Migraine pain results primarily from increased activity of several agents that regulate blood vessels and sensory function of the brain ${ }^{1}$. In about 15 percent of patients, migraine attacks may be accompanied by aura (visual, sensory, or language symptoms). Other accompanying symptoms may include photophobia (excessive sensitivity to light), phonophobia (fear of loud sounds), nausea or vomiting'.

Beta-adrenergic blockers, such as propranolol, are among the most prescribed drugs for migraine prophylaxis ${ }^{9,10}$. Propranolol has been prescribed for migraine prophylaxis since 1966 when Rabkin et al. discovered its effectiveness in migraine headache in their patients who were being treated for angina pectoris. There is clear evidence that propranolol is more effective than placebo in the treatment of migraine 9 . The usual propranolol doses for migraine prevention in clinical trials have ranged from 80 to $160 \mathrm{mg}$ a day ${ }^{9-12}$. In a clinical trial in BSMMU showed a reduction in $53.17 \%$ of baseline headache frequency and $15.16 \%$ of baseline pain severity ${ }^{13}$. Adverse events most commonly reported with beta-blockers are fatigue, depression, nausea, dizziness, and insomnia. These symptoms are fairly well tolerated and are seldom the cause of premature withdrawal. Antidepressants, especially tricyclic agents such as amitriptyline and nortriptyline, have also been a mainstay in the prophylatic therapy of migraine ${ }^{14}$. Amitriptyline is a mixed serotonergic and noradrenergic reuptake inhibitor with well-established efficacy in chronic pain relief and migraine prophylaxis ${ }^{15,16}$. It is useful for the treatment of patients with migraine and comorbid depression ${ }^{17}$. Common side effects of amitriptyline include dry mouth, constipation, and sedation. They may also cause slowing of atrioventricular conduction and orthostatic hypotension.

This study was intended to compare the efficacy and safety of propranolol and amitriptyline in prevention of migraine attack when used in combination.

\section{Methodology}

Study Settings and Population: This study was designed as single centre, parallel, randomized control trial. This study was conducted in headache clinic at Banghabandhu Sheikh Medical University (BSMMU), Dhaka, Bangladesh from July 2012 to June 2014 for a period of two (02) years. Migraine patients with or without aura of 16 to 50 years of age, patients not on any prophylactic medication and patients willing to take part in the study were included for this study. Age less than 16 years or more than 50 years, patients with chronic migraine, complicated migraine, ophthalmoplegic migraine, basilar migraine, catamenial migraine, patients on prophylactic medication, pregnant women, lactating mother, patients having history of bronchial asthma, cardiac arrhythmia, ischemic heart disease, bladder outlet obstruction or any known hypersensitivity to these drugs, patients with any serious co morbid condition such as uncontrolled hypertension, heart failure, hepatic or renal impairment, diabetes mellitus were excluded from this study. Informed written consent was taken from all patients. Migraine was diagnosed according to the criteria of the Headache Classification Committee of the International Headache Society, 2004 (IHS) ${ }^{22}$. Detailed history, general examination, neurological examination including fundoscopy and relevant systemic examination was done. Before the commencement of the study, the protocol for the following study was approved by ethical authority.

Randomization and Blinding: Patients meeting all the criteria wasrandomized for two (02) treatment groups designated as the group A who were treated with Amitriptyline and the group B who were treated with the combination of amitriptyline and propranolol.

Intervention: The doses of propranolol was $20 \mathrm{mg} \mathrm{BD}$ for the first two weeks, $20 \mathrm{mg}$ TDS for the next two weeks and finally $40 \mathrm{mg} \mathrm{BD}$ for the consecutive 8 weeks. The doses of amitriptyline was $10 \mathrm{mg}$ in the first two weeks and $25 \mathrm{mg}$ during second two weeks once at bedtime and $50 \mathrm{mg}$ at bed time in the next 8 weeks. The 
doses of each of these drugs wasthe same when given alone or in combination.

Follow up and Outcome Measures: Patients was followed for a three months period during which they wasinstructed to maintain a headache diary with the following information: presence of headache and intensity of headache by Visual Analogue Pain Scale. This wasalso include the need for analgesic for headache. Patients was asked to return on days 30,60 and 90. The primary outcome evaluated was the proportion of patients in each group that achieved a $50 \%$ reduction in the number of days with headache. Secondary outcomes was reduction of visual analogue pain scale score, the number of days with headache per month, frequency of side effects, and the proportion of patients abandoning the study before the end of medication. The causes of noncompliance and side effects was individually registered.

Statistical Analysis: After collection all the data were checked and edited. Then data were entered into the computer with the help of software SPSS for windows programmed version 16.0. After frequency run, data were cleaned and frequencies were checked. An analysis plan was developed keeping in view with the objectives of the study. Cross tabulation was prepared and a comparison had been made between, Data was presented as means (SD) and analyzed with 2-tailed $t$ tests when normally distributed. Every data was kept confidential.

\section{Results}

A clinical trial study was carried out to know Propranolol and Amitriptyline in combination more effective than monotherapy of amitriptyline in migraine prophylaxis. A total 80 adult patients were selected according to selection criteria. The patients were categorized in to 2 groups. Group-A received Amitribtyline and Group-B received combination drugs.

Table 1: Distribution of age among the patient

\begin{tabular}{lccc}
\hline Age Group & Group A & Group B & P value \\
\hline 16 to 25 Years & $24(60.0 \%)$ & $17(42.5 \%)$ & \\
26 to 35 Years & $14(35.0 \%)$ & $14(35.0 \%)$ & \\
36 Years and above & $2(5.0 \%)$ & $9(22.5 \%)$ & \\
Total & $\mathbf{4 0 ( 1 0 0 . 0 \% )}$ & $\mathbf{4 0}(\mathbf{1 0 0 . 0 \% )}$ & \\
\hline
\end{tabular}

Mean \pm SD (27.22 \pm 7.85$), \quad$ Min : 16, $\quad$ Max: 50

Gr-A Amitriptyline; Gr-B Combination

Table 1 shows that distribution of age among the patient. In group A, 16-25 age group were 24 (60.0\%),
26-35 age group were 14(35.0\%), 36 and above age group were $2(5.0 \%)$. In group B, 16-25 age group were $17(42.5 \%), 26-35$ age group were $14(35.0 \%), 36$ and above age group were $9(22.5 \%)$. The association was not statistically significant.

Table 2: Comparison of pain in Firstand Final follow up among the patients

\begin{tabular}{lcccc}
\hline Duration & \multicolumn{2}{c}{ Group A } & \multicolumn{2}{c}{ Group B } \\
\cline { 2 - 5 } & $\mathbf{1 s t}$ FU & Final FU & 1st FU & Final FU \\
\hline 1-4hours & $1(2.5 \%)$ & $24(60.0 \%)$ & $0(0.0)$ & $28(70.0 \%)$ \\
5-8 hours & $16(13.3 \%)$ & $14(35.0 \%)$ & $18(15.0)$ & $12(30.0 \%)$ \\
9-12hours & $14(35.0 \%)$ & $2(5.0 \%)$ & $21(52.5)$ & $0(0.0 \%)$ \\
$>13$ hours & $9(22.5 \%)$ & $0(0.0 \%)$ & $1(2.5)$ & $0(0.0 \%)$ \\
Total & $\mathbf{4 0 ( 1 0 0 . 0 \% )}$ & $\mathbf{4 0 ( 1 0 0 . 0 \% )}$ & $\mathbf{4 0 ( 1 0 0 . 0 \% )}$ & $\mathbf{4 0 ( 1 0 0 . 0 \% )}$ \\
P value & & & & \\
\hline
\end{tabular}

Gr-A Amitriptyline; Gr-B Combination

Table 2 shows that duration of pain found in during 1st visit among the patients. In group $\mathrm{A}$, duration of pain 1-4 hours 1 (2.5.0\%), 5-8 hours $16(13.3 \%)$ 9-12 hours $14(35.0 \%)$, Above. 13 hours $9(22.5 \%)$. In group B, duration of pain 1-4 hours $0(0.0), 5-8$ hours $18(15.0)$ 9-12 were 21(52.5), above 13 hours pain duration were $1(2.5 \%)$. The difference were not statistically significant. Duration of pain was recorded in final follow up among the patients. In group A, duration of pain 1-4 hours $24(60.0 \%), 5-8$ hours $14(35.0 \%), 9-12$ hours $2(5.0 \%)$. In group $\mathrm{B}$, duration of pain 1-4 hours $28(70.0 \%), 5-8$ hours $12(30.0 \%), 9-12$ hours were not found. The different was not statistically significant.

Table 3: Distribution of patients by side effects of drugs

\begin{tabular}{lccc}
\hline Adverse effects & Group A & Group B & P value \\
\hline No adverse effect & $26(65.0 \%)$ & $22(55.0 \%)$ & \\
Drowsiness & $6(15.0 \%)$ & $8(20.0 \%)$ & \\
Dry mouth & $6(15.0 \%)$ & $6(15.0 \%)$ & \\
Constipation & $2(5.0 \%)$ & $2(5.0 \%)$ & 0.0001 \\
Fatigue & $0(0.0 \%)$ & $2(5.0 \%)$ & \\
Bradycardia & $0(0.0 \%)$ & $0(0.0 \%)$ & \\
Total & $\mathbf{4 0 ( 1 0 0 . 0 \% )}$ & $\mathbf{4 0}(\mathbf{1 0 0 . 0} \%)$ & \\
\hline
\end{tabular}

Gr-A Amitriptyline; Gr-B Combination

Table 3 shows that the distribution of patients by side effects of drugs. In group A, no adverse effect was found $26(65.0 \%)$, drowsiness $6(15.0 \%)$, dryness of mouth $6(15.0 \%)$, constipation $2(5.0 \%)$, fatigue and bradycardia were not found. In group B, no adverse effect was found $22(55.0 \%)$, drowsiness $8(20.0 \%)$, dryness of mouth $6(15.0 \%)$, constipation $2(5.0 \%)$, fatigue $2(5.0 \%)$ and bradycardia were not found. 
Table 4: Number of attack and headache before treatment and subsequent follow up (Mean \pm SD)

\begin{tabular}{lccc}
\hline Variables & Group A & Group B & P value \\
\hline $\begin{array}{l}\text { Attack Before } \\
\text { Treatment }\end{array}$ & $5.825 \pm 2.18$ & $6.7 \pm 1.53$ & 0.085 \\
$\begin{array}{l}\text { Headache Before } \\
\text { Treatment }\end{array}$ & $7.625 \pm 2.50$ & $8.7 \pm 2.07$ & 0.094 \\
$\begin{array}{l}\text { Attach During First } \\
\text { Follow Up }\end{array}$ & $3.625 \pm 1.89$ & $4.425 \pm 1.31$ & 0.013 \\
$\begin{array}{l}\text { Headache During First } \\
\text { Follow Up }\end{array}$ & $4.675 \pm 2.23$ & $5.575 \pm 1.66$ & 0.037 \\
$\begin{array}{l}\text { Attach During Second } \\
\text { Follow Up }\end{array}$ & $2.475 \pm 1.67$ & $2.6 \pm 1.21$ & 0.002 \\
$\begin{array}{l}\text { Headache During } \\
\text { Second Follow Up }\end{array}$ & $2.875 \pm 2.11$ & $3.6 \pm 1.12$ & 0.001 \\
$\begin{array}{l}\text { Attach During Third } \\
\text { Follow Up }\end{array}$ & $1.675 \pm 1.09$ & $1.3 \pm .96$ & 0.000 \\
$\begin{array}{l}\text { Headache During } \\
\text { Third Follow Up }\end{array}$ & $2.35 \pm 1.64$ & $1.9 \pm 1.44$ & 0.005 \\
\hline $\begin{array}{l}\text { Gr-A Amitriptyline; Gr-B Combination } \\
\text { Non }\end{array}$ & &
\end{tabular}

Number of attack and headache before treatment and subsequent follow up with medication it was found that number of attach and headache gradually decrease. The differences are statistically significantly.

\section{Discussion}

The treatment of migraine involves both acute and preventive drugs and non-pharmacological strategies. Preventive treatment is necessary when the migraine attacks are unacceptably frequent, prolonged, severe, unresponsive to acute medication or associated with hemiparesis or prolonged aura. It is therefore designed to reduce the frequency, duration and/or severity of the attacks. In addition, preventive treatment often makes migraine attacks more responsive to acute migraine therapies, reduces migraine associated disability, improves the patients ability to function and decreases health care costs and use of healthcare resources ${ }^{16}$.

In this present study a total 120 adult patients were selected and according to selection criteria divided into two groups, Group-A received Amitriptyline, Group-B received combination drugs. In group $\mathrm{A}, 16-25$ age group were $24(60.0 \%), 26-35$ age group were $14(35.0 \%), 36$ and above age group were $2(5.0 \%)$. In group B, 16-25 age group were 17(42.5\%), 26-35 age group were $14(35.0 \%), 36$ and above age group were $9(22.5 \%)$. The mean age of study population was $27.22 \pm 7.85$ and their minimum and maximum age were 16 years and 60 years respectively. Similar result was reported ${ }^{17}$ and mentioned that migraines usually develop in childhood, adolescence or early adulthood. It has been documented that prevalence peak of migraine is at about age 40 and then prevalence declines progressively which is not headache intensity declined from 40 years to 74 years without change in headache frequency or headache duration which is consistent with the present study ${ }^{18-22}$.

In this study the duration of pain was recorded during the $1^{\text {st }}$ visit among the patients. In group A, duration of pain 1-4 hours $1(2.5 .0 \%)$, 5-8 hours $16(13.3 \%)$, 9-12 hours $14(35.0 \%)$ and above 13 hours $9(22.5 \%)$. In group $\mathrm{B}$, duration of pain 1-4 hours $0(0.0), 5-8$ hours 18(15.0) 9-12 were 21(52.5), above 13 hours pain duration were $1(2.5 \%)$. Duration of pain found in final follow up among the patients; in group A, duration of pain 1-4 hours 24(60.0\%), 5-8 hours 14(35.0\%), 9-12 hours $2(5.0 \%)$. In group B, duration of pain 1-4 hours $28(70.0 \%), 5-8$ hours $12(30.0 \%), 9-12$ hours were not found. There was no significant relationship between pain and migraine $(\mathrm{p}>0.05)$.

The side effects of drugs were recorded in this study. In group A, no adverse effect was found 26(65.0\%), drowsiness $6(15.0 \%)$, dryness of mouth $6(15.0 \%)$, constipation $2(5.0 \%)$, fatigue and bradycardia were not found. In group B, no adverse effect was found $22(55.0 \%)$, drowsiness $8(20.0 \%)$, dryness of mouth $6(15.0 \%)$, constipation $2(5.0 \%)$, fatigue $2(5.0 \%)$ and bradycardia were not found. In conclusion side effects are no adverse effect throughout the period of study.

Number of attack and headache before treatment and subsequent follow up with medication it was found that number of attach and headache gradually decrease. The differences are statistically significantly.

Although optimum care had been tried by the researcher in every steps of this study, still some limitations existed: The study was conducted in a selected area. So the study population might not represent the whole the people. Time and Budget constraints are the important reasons. In spite of maximum effort by the researcher due to time and resource limitation sample size was small; a larger sample size would have given a better result.

\section{Conclusion}

The study findings of this study shown that the efficacy of Propranolol and Amitriptyline in combination is more effective than monotherapy of Amitriptyline in migraine prophylaxis. The side effects are minimum than monotherapy. A large scale and multicenter study should be done to evaluate efficacy of Propranolol and Amitriptyline in combination is more effective than 
monotherapy of either drug in migraine prophylaxis.

\section{References}

1. Goadsby PJ, Raskin NH. Chapter 15. Headache. In: Fauci AS, Braunwald E, Kasper DL, Hauser SL, Longo DL, Jameson JL, et al., eds. Harrison's principles of internal medicine. 17th ed. New York: The McGraw-Hill Companies; 2008.

2. Silberstein SD. Preventive migraine treatment. NeurolClin. 2009 May;27(2):429-43. PMID 19289224.

3. Solomon GD, Santanello N. Impact of migraine and migraine therapy on productivity and quality of life. Neurology. 2000;55(9 Suppl 2):S29-35. PMID 11089517.

4. Lipton RB, Stewart WF, Diamond S, et al. Prevalence and burden of mi $\neg$ graine in the United States: data from the American Migraine Study II. Headache 2001;41:646-657.

5.Hannan MA et al, Study of epidemiological features of primary headache patients in a tertiary centre in Bangladesh. Bangladesh J of Neuroscience.2007;23(1);11-22.

6. Habib Mansur et al. Headache, study of 3350 cases. Bangladesh J Neuroscience. 2001; 17(1): 1-5.

7. Silberstein SD. Practice parameter: evidence-based guidelines for migraine headache (an evidence-based review): report of the Quality Standards Subcommittee of the American Academy of Neurology. Neurology 2000;55:754-762.

8. Brandes JL, Saper JR, Diamond M, et al. Topiramate for migraine prevention: a randomized controlled trial. JAMA 2004; 291:965-973.

9. Linde K, Rossnagel K. Propranolol for migraine prophylaxis. Cochrane Database Syst Rev 2004:CD003225.

10. Tvedskov JF, Thomsen LL, Thomsen LL, et al. The effect of propranolol on glyceryltrinitrate-induced headache and arterial response. Cephalalgia 2004;24:1076-1087.
11. Pradalier A, Serratrice G, Collard M, et al. Long-acting propranolol in migraine prophylaxis: results of a double-blind, placebo-controlled study. Cephalalgia 1989;9:247-253.

12. al-Qassab HK, Findley LJ. Comparison of propranolol LA 80 $\mathrm{mg}$ and propranolol LA $160 \mathrm{mg}$ in migraine prophylaxis: a placebo controlled study. Cephalalgia 1993;13:128-131.

13. Chowdhury MI, Study on propranolol vs sodium valproate in the prevention of migraine, Dept. of Neurology, BSMMU,2006

14. Punay NC, Couch JR. Antidepressants in the treatment of migraine headache. Curr Pain Headache Rep 2003;7:51-54.

15. Ramadan NM. Prophylactic migraine therapy: mechanisms and evidence. Curr Pain Headache Rep 2004;8:91-5.

16 Bigal ME, Krymchantowski AV, Rapoport AM. Prophylactic migraine therapy: emerging treatment options. Curr Pain Headache Rep 2004;8:178-84.

17 Campo-Arias A. Antidepressants in migraine prophylaxis: an approximation. Rev Neurol 2004;38:864-8.

18. Bordini CA, Arruda MA, Ciciarelli MC, Speciali JG. Propranolol vsflu $\neg$ narizinevsflunarizine plus propranolol in migraine without aura pro $\neg$ phylaxis: a double-blind trial. ArqNeuropsiquiatr 1997;55:536-341.

19. Kaniecki RG. A comparison of divalproex with propranolol and placebo for the prophylaxis of migraine without aura. Arch Neurol 1997;54:1141-1145.

20. Keskinbora K, Aydinli I. A double-blind randomized controlled trial of topiramate and amitriptyline either alone or in combination for the prevention of migraine. ClinNeurolNeurosurg 2008;110:979-984.

21. Peterlin BL, Calhoun AH, Siegel S, Mathew NT. Rational combination therapy in refractory migraine. Headache 2008;48:805-819.

22. Headache Classification Committee of the International Headache Society, 2004. 